\title{
Switching Times and Transition Widths in Digital Recording Theory
}

\author{
B. K. Middleton, Member, IEEE, J. J. Miles, Member, IEEE, and M. M. Aziz
}

\begin{abstract}
Calculations are carried out to show the effects of finite switching times of head fields and of recording media on recorded transition widths. In both cases the transition widths are increased and these effects are captured in simple analytical expressions.
\end{abstract}

Index Terms-Switching times.

\section{INTRODUCTION}

$\mathbf{R}$ ECORDING at very high data rates will eventually suffer from limitations imposed by the rates at which head fields can be changed and the rates at which media can respond. This paper deals with the influence of both of these factors on the nature of the recorded distributions in thin film recording media.

\section{THEORY}

\section{A. Record Head Field Switching}

The longitudinal component of the record head field is assumed to take the form

$$
H_{x}=i(t) \cdot \frac{y}{y^{2}+x^{2}}=i(t) \cdot \frac{y}{y^{2}+\left(x^{\prime}+v t\right)^{2}}
$$

where

$$
\begin{aligned}
& i(t) \quad \text { is the record current, } \\
& t \quad \text { is time, } \\
& x, x^{\prime}, \text { and } y \text { are the coordinates defined in Fig. 1, and } \\
& v \quad \text { is medium velocity. }
\end{aligned}
$$

$i(t)$ is assumed to take the form of a ramp function, namely

$$
i(t)=I_{o} f(t)
$$

where

$$
f(t)= \begin{cases}t / T & t<T \\ \pm 1 & |t|>T .\end{cases}
$$

$T \quad$ is the field rise time,

$I_{o}=2 H_{c} y$ is the peak current value, and

$H_{c} \quad$ is the recording medium coercivity.

The form that $f(t)$ takes in practical systems depends on the way heads are driven and the circuitry used for that purpose. In our case a ramp is a reasonable approximation although other forms

Manuscript received October 11, 2000.

The work of M. M. Aziz was sponsored by the Engineering and Physical Sciences Research Council.

B. K. Middleton and J. J. Miles are with the Department of Computer Science, University of Manchester, Manchester M13 9PL, England (e-mail: B.Middleton@cs.man.ac.uk; J.Miles@man.ac.uk).

M. M. Aziz was with the Department of Computer Science, University of Manchester, Manchester M13 9PL, England, and is now with the University of Exeter, Exeter EX4 4QF, England (e-mail: M.M.Aziz@exeter.ac.uk).

Publisher Item Identifier S 0018-9464(01)05619-9.

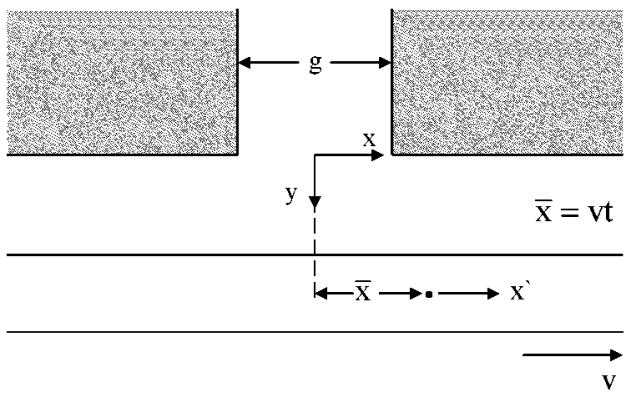

Fig. 1. Definition of parameters during recording.

have been tried elsewhere [1], [2]. An advantage of the ramp is that it is amenable to algebraic analysis and avoids purely numerical solutions [1], [2].

The time $t_{o}$ at which a location $x^{\prime}$ in the recording medium experiences the highest field is found by solving $d H_{x} / d t=0$. This leads to

$$
v t_{O}=\sqrt{x^{2}+y^{2}} \quad t_{O}<T .
$$

Using (3) in (1) the maximum field experienced by each element $x^{\prime}$ of the medium is

$$
H_{x}=\frac{H_{c} y^{2}}{v T} \cdot \frac{1}{x^{\prime}+\sqrt{x^{\prime 2}+y^{2}}} .
$$

Equation (4) is now taken as the effective head field. Although this field develops over time and is not instantaneously applied, as is usually the case, it is taken as if it is. This then allows a straight forward development of record theory with switching times included.

The center of the transition is at $x_{o}^{\prime}$ where the head field is equal to the coercivity, $H_{c}$, and is given by

$$
x_{o}^{\prime}=\frac{y^{2}}{2 v T}-\frac{v T}{2} .
$$

At this point the field gradient is easily shown to be

$$
\frac{d H_{x}}{d x^{\prime}}=-\frac{H_{c}}{y} \cdot\left(\frac{2(v T / y)}{1+(v T / y)^{2}}\right) \quad v T>y .
$$

This can be written as

$$
\frac{d H_{x}}{d x^{\prime}}=-\frac{H_{c}}{y} \cdot F
$$

where

$$
F= \begin{cases}\frac{2(v T / y)}{1+(v T / y)^{2}} & v T>y \\ 1 & v T<y\end{cases}
$$

Equation (7) is the usual expression for head field gradient, i.e., $-H_{c} / y$ when $F=1$, but when $v T>y$ this is multiplied by 


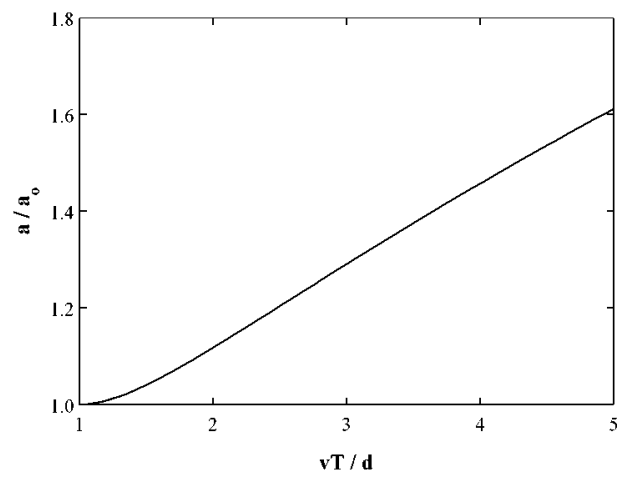

Fig. 2. Variation of reduced transition width as a function of reduced switching time.

an additional term which accounts for the switching time of the record current.

Simple modifications can now be made to many of the earlier calculations of the transition widths in longitudinal recording especially those of Williams and Comstock [3]. Here a modification of the approach of Middleton [4] for thin films yields

$$
a=\frac{M_{r} d}{\pi \chi H_{c} F}+\sqrt{\left(\frac{M_{r} d}{\pi \chi H_{c} F}\right)^{2}+\frac{M_{r} d \delta}{\pi H_{c} F}}
$$

where

$\chi \quad$ is the slope of the hysteresis loop of the recording medium at $H=H_{c}$,

$M_{r} \quad$ is the remanent magnetization, and

$y \quad$ takes the value of the head to medium spacing $d$, and

$\delta \quad$ is the thickness of the recording medium.

When $\chi=\infty$ the transition width becomes

$$
a=\sqrt{\left(\frac{M_{r} d \delta}{\pi H_{c}} \cdot \frac{1+(v T / d)^{2}}{2(v T / d)}\right)} \quad v T>d .
$$

A graph of $\left(a / a_{o}\right)$ where $a_{o}=\sqrt{M_{r} \delta d / \pi H_{c}}$, as a function of $(v T / d)$ is shown in Fig. 2 where the effect of finite $T$ is clearly shown to increase the transition widths.

Equations (9) and (10) apply for $d>\delta$ but if this condition is not met $d$ should be replaced by $(d+\delta / 2)$.

In perpendicular recording the transition widths previously derived [4] can also be modified to take into account the finite rise times in the recording head fields. When recording is achieved by a single pole head and its perpendicular field and gradient take the same form as those of the longitudinal components of a ring head the transition width becomes, for $M_{r}>H_{c}$ [4]

$$
\begin{gathered}
a=\frac{2}{\pi}(d+\delta / 2)\left[\frac{1+(v T /(d+\delta / 2))^{2}}{2(v T /(d+\delta / 2))}\right]-\frac{\delta}{2} \\
v T>(d+\delta / 2) .
\end{gathered}
$$

Again as $T$ increase so does the transition width.

\section{B. Switching of Recording Media-I}

To study the switching of a thin film recording medium the record head field is assumed to be suddenly applied and take the form

$$
H_{x}=2 H_{c} \cdot \frac{y^{2}}{y^{2}+\left(x^{\prime}+v t\right)^{2}} .
$$

As the medium moves through this field the magnetization at the point $x^{\prime}$ will depend on the time spent in the head field and its magnitude. It can be calculated using [5]

$$
M=-M_{r}+\int_{t_{1}}^{t_{2}} 2 M_{r} s\left(H-H_{o}\right) d t
$$

where

$s \quad$ is the switching constant [5],

$H_{O} \quad$ is a constant of the switching process [5],

$t_{1}=0 \quad$ is the time at which the head field is applied, and

$t_{2}=\quad$ is the time for the point $x^{\prime}$ to move to $y$ where-

$\left(y-x^{\prime}\right) / v$ upon the head field is equal to the coercivity and changes of magnetization cease.

Evaluation of (13) for the head field (12) leads to

$$
M=-M_{r}+\frac{2 M_{r} s}{v} \cdot\left(2 H_{c} y \cdot\left(\frac{\pi}{4}-\tan ^{-1} \frac{x^{\prime}}{y}\right)-H_{o} y+H_{\circ} x^{\prime}\right)
$$

and therefore

$$
\frac{d M}{d x^{\prime}}=\frac{2 M_{r} s}{v}\left(-2 H_{c} y \cdot \frac{y}{y^{2}+x^{\prime 2}}+H_{o}\right) .
$$

The center of the transition $x_{o}^{\prime}$ is at $M=0$ and using $x_{o}^{\prime}<y$, so that $\tan ^{-1}\left(x^{\prime} / y\right)$ can be replaced by $\left(x^{\prime} / y\right)$, it is easily shown from (14) that

$$
x_{o}^{\prime}=y\left(\frac{\pi}{2}-1\right)-\frac{v}{2 H_{c} s} .
$$

Substitution of this into (15) gives the head field gradient at the middle of the transition. Equating this to $\left(2 M_{r} / \pi a\right)$ and putting $y=d$ produces a transition width parameter $a$ of

$$
a=\frac{v}{\pi s H_{c}} \cdot\left(\frac{2}{1+\left[(\pi / 2-1)^{2}-\left(v / 2 s H_{c} d\right)\right]^{2}}-1\right)^{-1} .
$$

Fig. 3 shows $a / d$, calculated using (17), plotted as a function of $v / 2 s H_{c} d$. This represents the minimum width that a transition could possibly take when the medium behavior is limited by its switching characteristics.

Again, the above equations apply for $d>\delta$ but if this condition is not met $d$ should be replaced by $(d+\delta / 2)$.

\section{Switching of Recording Media-II}

For another derivation of transition width consider the ideal case of a head field of the Karlqvist type at $y=0$. This has a constant amplitude $2 H_{c}$ under the gap and is zero elsewhere. Any element of medium passing under the head gap must be in its field for a minimum time $T$, determined from (13), if the magnetization is to be fully reversed and a complete transition is to be recorded. The corresponding distance the medium moves 


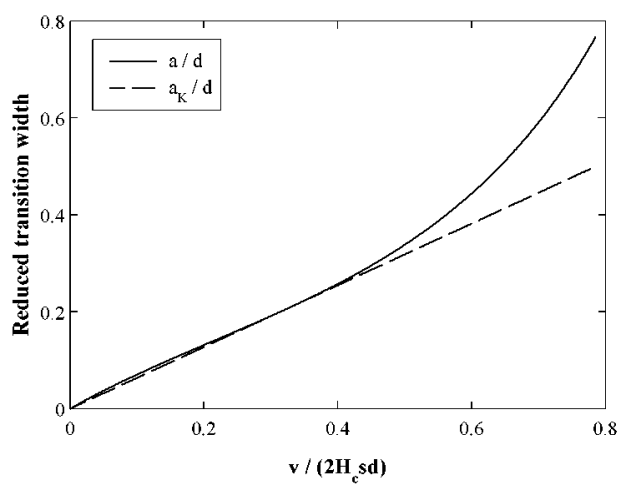

Fig. 3. Variation of reduced transition widths as a function of reduced medium velocity.

in this time is $v T$ and this is the transition width. Equating this to $\pi a$, and putting $H_{o}=H_{c}$ [5], leads to

$$
a_{K}=v / \pi s H_{c}
$$

which is the minimum transition width parameter that can be recorded by this head. Equation (18) is represented by the solid line in Fig. 3.

If the head gap $g<v T$ the medium will have been in the head field for insufficient time for the magnetization to change state from $-M_{r}$ to $+M_{r}$ and so the recorded transition will not separate regions magnetized to their remanent values.

\section{DISCUSSION}

When recording is subject to a record head field which has a finite switching time the calculations have shown that there is no influence on transition width as long as the switching time
$T<d / v$. When this condition is not met then there will be increases in transition widths and therefore some deterioration of recording performance. The increases in transition width in longitudinal recording are shown in Fig. 2 and are broadly in line with those produced earlier by numerical means [1], [2].

When recording takes place on a medium which needs a finite time to change its magnetization state there is another minimum to the transition width which can be recorded. This is shown in Fig. 3. It is interesting to note that the calculated transition widths arising from different field distributions are close together over a wide range of switching constants.

\section{CONCLUSIONS}

Expressions have been derived which show the effects of finite switching times of head fields and recording media. In both cases finite switching times are deleterious and the largest of the transition widths cited represents an absolute minimum that could possibly be recorded.

\section{REFERENCES}

[1] P. Thayamballi, "Modeling the effects of write field rise time on the recording properties in thin film media," IEEE Trans. Magn., vol. 32, no. 1, pp. 61-66, Jan. 1996.

[2] X. Xing and H. N. Bertram, "Analysis of the effect of write head field rise time on signal, noise and nonlinear transition shift," J. Appl. Phys., vol. 85 , no. 8, pp. 5861-5863, Apr. 1999.

[3] M. L. Williams and R. L. Comstock, "An analytical model of the write process in digital magnetic recording," in 17th Annu., AIP Conf. Proc., Part 1, no. 5, 1971, pp. 738-742.

[4] B. K. Middleton, "Recording and reproducing processes," in Magnetic Recording Handbook, C. D. Mee and E. D. Daniel, Eds. New York, NY: McGraw-Hill, 1989, ch. 2, pp. 24-100.

[5] B. K. Middleton and J. J. Miles, "Fast switching and coercivity in thin film recording media," in Intermag 2001. 